\title{
Continuous dependence of semilinear Petrovsky equation
}

\author{
Hüseyin Kocaman, Metin Yaman*, Şevket Gür \\ Department of Mathematics, Sakarya University, Sakarya, Turkey. \\ Communicated by W. Shatanawi
}

\begin{abstract}
In this study, we obtain the continuous dependence on the coefficients of solutions of semilinear Petrovsky equation. Such models are involved in various fields of mathematical physics likewise geophysical and oceanic applications. (C)2017 All rights reserved.
\end{abstract}

Keywords: Semilinear Petrovsky equation, continuous dependence.

2010 MSC: 35B30, 35L35.

\section{Introduction}

In this paper we study the continuous dependence of semilinear Petrovsky equation.

$$
\begin{gathered}
\mathfrak{u}_{t t}+\Delta^{2} u+a u_{t}\left|u_{t}\right|^{p-2}+b u|u|^{q-2}=0, \\
u(x, 0)=u_{0}(x), \quad u_{t}(x, 0)=u_{1}(x) \text { in } \Omega, \\
u(x, t)=\Delta u=0 \quad \text { on }[0, T] \times \partial \Omega,
\end{gathered}
$$

where $a, b>0, \Omega \subset R^{n}$ is a bounded region with a smooth boundary $\partial \Omega, 2 \leqslant p<\infty, 2<q<\infty$ if $n=1,2$ and $2 \leqslant p \leqslant \frac{2(n-1)}{n-2}, 2<q \leqslant \frac{2(n-1)}{n-2}$ if $n \geqslant 3$.

The subject of continuous dependence of solutions of hyperbolic type problems in partial differential equations on the coefficients in the equations has been extensively studied in the last decades. The question of continuous dependence means, one wishes to know whether a small changes in the coefficients or the parameters will inference a small changes in the solutions of the problem.

In [4], the authors studied multidimensional marine riser equations:

$$
u_{t t}+k \Delta^{2} u+a \Delta u+\vec{g} \cdot \nabla u_{t}+b\left|u_{t}\right|^{p} u_{t}=0, x \in \Omega, t>0 .
$$

They obtained continuous dependence of the parameters a (coriolis force), $b$ (drag force), and $g$ (effective tension).

\footnotetext{
*Corresponding author

Email address: myaman@sakarya.edu.tr (Metin Yaman)
}

doi:10.22436/jnsa.010.05.33 
In the following problem, it is studied the continuous dependence of solutions for the damped nonlinear hyperbolic equation [15]:

$$
u_{t t}+\alpha \Delta^{2} u+\beta \Delta^{2} u_{t}+\Delta g(\Delta u)=0, \quad x \in \Omega, t>0 .
$$

Some relevant papers on continuous dependence problems on hyperbolic type are [5, 7, 12].

In 1990, You [16] considered energy decay rate and controllability for the Petrovsky equation in a higher dimensional bounded domain with homogeneous boundary conditions. Chen and Zhou [6] considered a semilinear Petrovsky equation with damping and source terms and they proved the solution blows up in finite time for suitable condition.

In [2] the authors considered the nonlinear damped semilinear Petrovsky equation

$$
u^{\prime \prime}-\Delta_{x}^{2} u+g\left(u^{\prime}\right)=b u|u|^{p-2}
$$

and proved the global existence of its solutions with the Faedo Galerkin procedure. In addition, they studied the asymptotic behavior of solutions. In [8], Han and Wang investigated asymptotic behavior for the solution of the Petrovsky equation with locally distributed damping. In [11], the authors considered the following Petrovsky equations:

$$
\begin{aligned}
u_{t t}+\Delta^{2} u+\left|u_{t}\right|^{p-1} u_{t} & =F_{u}(u, v), \\
v_{t t}+\Delta^{2} v+\left|v_{t}\right|^{p-1} v_{t} & =F_{v}(u, v),
\end{aligned}
$$

and investigated global existence, uniform decay and blow up solutions. In [14], Tahamtani and Shahrouzi considered the following semilinear Petrovsky equation

$$
u_{t t}+\Delta^{2} u-\int_{0}^{t} g(t-s) \Delta^{2} u(s) d s=|u|^{p} u
$$

and proved the existence of weak solutions. We also refer to the papers $[9,10,13,17]$ on the semilinear Petrovsky equation. Some papers about continuous dependence results can be seen in $[1,3]$. Since there is no study on the continuous dependence of Petrovsky equation, our goal is to investigate the continuous dependence of solutions to the problem (1.1)-(1.3) on the coefficients $a$ and $b$.

Lemma 1.1 (Sobolev-Poincaré inequality). Let $r$ be a number with $2 \leqslant r<\infty(n=1,2)$ or $2 \leqslant r \leqslant \frac{2(n-1)}{n-2}$ $(\mathrm{n} \geqslant 3)$, then there is a constant $\mathrm{d}=\mathrm{d}(\Omega, \mathrm{r})$ such that

$$
\|u\|_{r} \leqslant \mathrm{~d}\|\Delta \mathrm{u}\|_{2}
$$

for $u \in \mathrm{H}_{0}^{2}(\Omega)$.

\section{Priori estimates}

Theorem 2.1. Let $\mathrm{u}_{0} \in \mathrm{H}_{0}^{1}(\Omega)$ and $\mathrm{u}_{1} \in \mathrm{L}^{2}(\Omega)$. Then the solution $\mathrm{u}$ of the problem (1.1)-(1.3) satisfies the following estimates:

$$
\left\|u_{\mathrm{t}}(\mathrm{t})\right\|^{2} \leqslant \mathrm{D}_{1}, \quad\|\Delta \mathrm{u}\|^{2} \leqslant \mathrm{D}_{1}, \quad \text { and } \quad\|\mathrm{u}\|_{\mathrm{q}}^{\mathrm{q}} \leqslant \mathrm{D}_{2},
$$

where $\mathrm{D}_{1}$ and $\mathrm{D}_{2}$ are positive constants depending on the initial data and the parameters of (1.1).

Proof. If the equation (1.1) is multiplied by $u_{t}$ in $L_{2}(\Omega)$, then

$$
\frac{\mathrm{d}}{\mathrm{dt}}\left[\frac{1}{2}\left\|\mathrm{u}_{\mathrm{t}}\right\|^{2}+\frac{1}{2}\|\Delta \mathrm{u}\|^{2}+\frac{\mathrm{b}}{\mathrm{q}}\|\mathrm{u}\|_{\mathrm{q}}^{\mathrm{q}}\right]+\mathrm{a}\left\|\mathrm{u}_{\mathrm{t}}\right\|_{\mathrm{p}}^{\mathrm{p}}=0
$$


is obtained. Let $E_{1}(t)=\frac{1}{2}\left\|u_{t}\right\|^{2}+\frac{1}{2}\|\Delta u\|^{2}+\frac{b}{q}\|u\|_{q}^{q}$. Thus, from the equation (2.2) we have

$$
\frac{d}{d t} E_{1}(t) \leqslant 0
$$

and

$$
E_{1}(t) \leqslant E_{1}(0)
$$

The inequality (2.3) gives us all estimates in (2.1).

Theorem 2.2. Let $\mathfrak{u}_{1} \in \mathrm{H}^{2}(\Omega) \cap \mathrm{H}_{0}^{1}(\Omega)$. Then the solution $\mathrm{u}$ of the problem (1.1)-(1.3) satisfies the following estimates

$$
\left\|u_{t t}\right\|^{2} \leqslant D_{4} e^{D_{3} t}, \quad\left\|\Delta u_{t}\right\|^{2} \leqslant D_{4} e^{D_{3} t},
$$

where $\mathrm{D}_{3}$ and $\mathrm{D}_{4}$ are positive constants depending on the initial data and the parameters of (1.1).

Proof. We differentiate the equation (1.1) with respect to $t$

$$
u_{t t t}+\Delta^{2} u_{t}+a(p-1)\left|u_{t}\right|^{p-2} u_{t t}+b(q-1)|u|^{q-2} u_{t}=0 .
$$

We multiply the equation (2.5) by $u_{t t}$ in $L_{2}(\Omega)$, then we get

$$
\frac{d}{d t} E_{2}(t)+a(p-1) \int_{\Omega}\left|u_{t}\right|^{p-2} u_{t t}^{2} d x+b(q-1) \int_{\Omega}|u|^{q-2} u_{t} u_{t t} d x=0,
$$

where $E_{2}(t)=\frac{1}{2}\left\|u_{t}\right\|^{2}+\frac{1}{2}\left\|\Delta u_{t}\right\|^{2}$. Then from the equation (2.6) we have the following inequality

$$
\frac{d}{d t} E_{2}(t) \leqslant\left. b(q-1)\left|\int_{\Omega}\right| u\right|^{q-2} u_{t} u_{t t} d x \mid .
$$

Using Hölder and Sobolev-Poincaré inequalities and the estimate (2.1) we obtain the inequality

$$
\left.\left|b(q-1) \int_{\Omega}\right| u\right|^{q-2} u_{t} u_{t t} d x \mid \leqslant b(q-1)\|u\|_{(q-2) n}^{q-2}\left\|u_{t t}\right\|\left\|u_{t}\right\|_{\frac{2 n}{n-2}} \leqslant D_{3}\left\|u_{t t}\right\|\left\|\Delta u_{t}\right\|,
$$

where $D_{3}=b d(q-1)\left(D_{1}\right)^{\frac{q-2}{2}}$. Apply the Cauchy inequality on the right hand side of the inequality (2.8) and rewrite (2.7) to get

$$
\frac{d}{d t} E_{2}(t) \leqslant D_{3} E_{2}(t)
$$

The last inequality gives the estimate

$$
E_{2}(t) \leqslant e^{D_{3} t} E_{2}(0) .
$$

Hence, proof is completed.

\section{Continuous dependence on parameters}

3.1. Continuous dependence on the coefficient a

Assume that $u$ is the solution of the problem

$$
\begin{gathered}
u_{t t}+\Delta^{2} u+a u_{t}\left|u_{t}\right|^{p-2}+b u|u|^{q-2}=0, \\
u(x, 0)=u_{0}(x), \quad u_{t}(x, 0)=u_{1}(x) \quad \text { in } \Omega,
\end{gathered}
$$




$$
u(x, t)=\Delta u=0 \quad \text { on }[0, T] \times \partial \Omega .
$$

Assume that $v$ is the solution of the problem

$$
\begin{gathered}
v_{\mathrm{tt}}+\Delta^{2} v+(\mathrm{a}+\alpha) v_{\mathrm{t}}\left|v_{\mathrm{t}}\right|^{\mathrm{p}-2}+\mathrm{b} v|v|^{\mathrm{q}-2}=0, \\
v(\mathrm{x}, 0)=\mathrm{u}_{0}(\mathrm{x}), \quad v_{\mathrm{t}}(\mathrm{x}, 0)=\mathrm{u}_{1}(\mathrm{x}) \quad \text { in } \Omega, \\
v(\mathrm{x}, \mathrm{t})=\Delta v=0 \quad \text { on }[0, \mathrm{~T}] \times \partial \Omega,
\end{gathered}
$$

and $w=u-v$ is the solution of the problem

$$
\begin{gathered}
w_{\mathrm{tt}}+\Delta^{2} w+a\left(\left|u_{\mathrm{t}}\right|^{p-2} u_{\mathrm{t}}-\left|v_{\mathrm{t}}\right|^{p-2} v_{\mathrm{t}}\right)-\alpha\left|v_{\mathrm{t}}\right|^{p-2} v_{\mathrm{t}}+\mathrm{b}\left(|\mathrm{u}|^{\mathrm{q}-2} \mathrm{u}-|v|^{\mathrm{q}-2} v\right)=0, \\
w(x, 0)=0, \quad w_{\mathrm{t}}(x, 0)=0 \quad \text { in } \Omega, \\
w(x, \mathrm{t})=\Delta w=0 \quad \text { on }[0, \mathrm{~T}] \times \partial \Omega .
\end{gathered}
$$

Theorem 3.1. If $w$ is the solution of the problem (3.1)-(3.3), then

$$
\|\Delta w\| \rightarrow 0 \quad \text { as } \quad \alpha \rightarrow 0 .
$$

Proof. We multiply the equation (3.1) by $w_{t}$ in $L_{2}(\Omega)$ to obtain

$$
\begin{aligned}
& \frac{\mathrm{d}}{\mathrm{dt}} \mathrm{E}_{3}(\mathrm{t})+\mathrm{a} \int_{\Omega}\left(\left|u_{\mathrm{t}}\right|^{p-2} u_{\mathrm{t}}-\left|v_{\mathrm{t}}\right|^{p-2} v_{\mathrm{t}}\right) w_{\mathrm{t}} \mathrm{d} x \\
& -\alpha \int_{\Omega}\left|v_{\mathrm{t}}\right|^{p-2} v_{\mathrm{t}} w_{\mathrm{t}} \mathrm{d} x+\mathrm{b} \int_{\Omega}\left(|\mathrm{u}|^{q-2} u-|v|^{q-2} v\right) w_{\mathrm{t}} \mathrm{d} x=0,
\end{aligned}
$$

where $E_{3}(t)=\frac{1}{2}\left\|w_{t}\right\|^{2}+\frac{1}{2}\|\Delta w\|^{2}$. Since $a \int_{\Omega}\left(\left|u_{t}\right|^{p-2} u_{t}-\left|v_{t}\right|^{p-2} v_{t}\right) w_{t} d x>0$, then we get from the relation (3.4)

$$
\frac{d}{d t} E_{3}(t) \leqslant\left.\left|\alpha \int_{\Omega}\right| v_{t}\right|^{p-2} v_{t} w_{t} d x|+| b \int_{\Omega}\left(|u|^{q-2} u-|v|^{q-2} v\right) w_{t} d x \mid .
$$

Now, we evaluate first term on the right hand side of (3.5)

$$
\left.\left.\left|\alpha \int_{\Omega}\right| v_{\mathrm{t}}\right|^{p-2} v_{\mathrm{t}} w_{\mathrm{t}} \mathrm{d} x|\leqslant| \alpha\left|\int_{\Omega}\right| v_{\mathrm{t}}\right|^{p-1}\left|w_{\mathrm{t}}\right| \mathrm{d} x \leqslant|\alpha|\left\|w_{\mathrm{t}}\right\|\left\|v_{\mathrm{t}}\right\|_{2(\mathrm{p}-1)}^{p-1} \leqslant \frac{1}{2}\left\|w_{\mathrm{t}}\right\|^{2}+\frac{\alpha^{2}}{2}\left\|v_{\mathrm{t}}\right\|_{2(\mathrm{p}-1)}^{2(p-1)} .
$$

Using the Sobolev-Poincaré inequality we obtain from (3.6)

$$
\left.\left|\alpha \int_{\Omega}\right| v_{\mathrm{t}}\right|^{p-2} v_{\mathrm{t}} w_{\mathrm{t}} \mathrm{d} x \mid \leqslant \frac{1}{2}\left\|w_{\mathrm{t}}\right\|^{2}+\frac{\alpha^{2}}{2} \mathrm{~d}\left\|\Delta v_{\mathrm{t}}\right\|^{2(\mathrm{p}-1)},
$$

where $d$ is the Sobolev constant. If we use the mean value theorem and Hölder's inequality to the second term on the right hand side of (3.5), then we have

$$
\begin{aligned}
& \left|b \int_{\Omega}\left(|u|^{\mathbf{q}-2} u-|v|^{\mathbf{q}-2} v\right) w_{\mathrm{t}} \mathrm{d} x\right| \leqslant \mathrm{b}(\mathbf{q}-1) \int_{\Omega}\left(|\mathbf{u}|^{\mathbf{q}-2}+|v|^{\mathbf{q}-2}\right)|w|\left|w_{\mathrm{t}}\right| \mathrm{d} x \\
& \leqslant b(q-1)\|w\|_{\frac{2 n}{n-2}}\left\|w_{t}\right\|\left(\|u\|_{(q-2) n}^{q-2}+\|v\|_{(q-2) n}^{q-2}\right) .
\end{aligned}
$$


We use the Sobolev inequality in (3.8) to get

$$
\left|\mathrm{b} \int_{\Omega}\left(|\mathbf{u}|^{\mathbf{q}-2} \mathbf{u}-|v|^{\mathbf{q}-2} v\right) w_{\mathrm{t}} \mathrm{dx}\right| \leqslant \mathrm{b}(\mathbf{q}-1) \mathrm{d}^{2}\|\Delta w\|\left\|w_{\mathrm{t}}\right\|\left(\|\Delta \mathbf{u}\|^{\mathbf{q}-2}+\|\Delta v\|^{\mathbf{q}-2}\right) .
$$

Hence using the estimate (2.1) in the inequality (3.9) we get

$$
\left|\mathrm{b} \int_{\Omega}\left(|u|^{q-2} u-|v|^{q-2} v\right) w_{\mathrm{t}} \mathrm{dx}\right| \leqslant D_{5}\|\Delta w\|\left\|w_{\mathrm{t}}\right\| \leqslant \frac{D_{5}}{2}\|\Delta w\|^{2}+\frac{\mathrm{D}_{5}}{2}\left\|w_{\mathrm{t}}\right\|^{2},
$$

where $D_{5}=2 b(q-1) d^{2}\left(D_{1}\right)^{\frac{q-2}{2}}$. The estimates (2.4), (3.5), (3.7), and (3.10) give us the following differential inequality

$$
\frac{d}{d t} E_{3}(t) \leqslant M_{3} E_{3}(t)+K_{1} \alpha^{2} e^{K_{2} t},
$$

where $M_{3}=1+D_{5}, K_{1}=\frac{d}{2} D_{4}^{p-1}, K_{2}=D_{3}(p-1)$. Thus the inequality (3.11) concludes the estimate

$$
E_{3}(t) \leqslant \frac{K_{1}}{K_{2}} e^{K_{3} t} \alpha^{2}
$$

where $\mathrm{K}_{3}=\mathrm{K}_{2}+\mathrm{M}_{3}$. Therefore we have $\|\Delta w\| \rightarrow 0$ as $\alpha \rightarrow 0$.

3.2. Continuous dependence on the coefficient $b$

Assume that $u$ is the solution of the problem

$$
\begin{gathered}
u_{t t}+\Delta^{2} u+a u_{t}\left|u_{t}\right|^{p-2}+b u|u|^{q-2}=0, \\
u(x, 0)=u_{0}(x), \quad u_{t}(x, 0)=u_{1}(x) \text { in } \Omega, \\
u(x, t)=\Delta u=0 \quad \text { on }[0, T] \times \partial \Omega .
\end{gathered}
$$

Assume that $v$ is the solution of the problem

$$
\begin{gathered}
v_{\mathrm{tt}}+\Delta^{2} v+a v_{\mathrm{t}}\left|v_{\mathrm{t}}\right|^{p-2}+(\mathrm{b}+\beta) v|v|^{\mathrm{q}-2}=0, \\
v(x, 0)=\mathrm{u}_{0}(\mathrm{x}), \quad v_{\mathrm{t}}(x, 0)=\mathrm{u}_{1}(\mathrm{x}) \text { in } \Omega, \\
v(x, \mathrm{t})=\Delta v=0 \quad \text { on }[0, \mathrm{~T}] \times \partial \Omega,
\end{gathered}
$$

and $w=u-v$ is the solution of the problem

$$
\begin{gathered}
w_{\mathrm{tt}}+\Delta^{2} w+a\left(\left|u_{\mathrm{t}}\right|^{p-2} u_{\mathrm{t}}-\left|v_{\mathrm{t}}\right|^{\mathrm{p}-2} v_{\mathrm{t}}\right)+\mathrm{b}\left(|\mathrm{u}|^{\mathrm{q}-2} \mathrm{u}-|v|^{\mathrm{q}-2} v\right)-\beta|v|^{\mathrm{q}-2} v=0, \\
w(x, 0)=0, \quad w_{\mathrm{t}}(\mathrm{x}, 0)=0 \quad \text { in } \Omega, \\
w(x, \mathrm{t})=\Delta w=0 \quad \text { on }[0, \mathrm{~T}] \times \partial \Omega .
\end{gathered}
$$

Theorem 3.2. If $w$ is the solution of the problem (3.13)-(3.15), then

$$
\|\Delta w\| \rightarrow 0 \quad \text { as } \beta \rightarrow 0 .
$$

Proof. We multiply the equation (3.12) by $w_{t}$ in $\mathrm{L}_{2}(\Omega)$ to get

$$
\begin{aligned}
& \frac{\mathrm{d}}{\mathrm{dt}} \mathrm{E}_{3}(\mathrm{t})+\mathrm{a} \int_{\Omega}\left(\left|u_{\mathrm{t}}\right|^{p-2} u_{\mathrm{t}}-\left|v_{\mathrm{t}}\right|^{p-2} v_{\mathrm{t}}\right) w_{\mathrm{t}} \mathrm{dx} \\
& -\beta \int_{\Omega}|v|^{\mathrm{q}-2} v w_{\mathrm{t}} \mathrm{d} x+\mathrm{b} \int_{\Omega}\left(|\mathrm{u}|^{\mathrm{q}-2} u-|v|^{\mathrm{q}-2} v\right) w_{\mathrm{t}} \mathrm{d} x=0 .
\end{aligned}
$$


By using the inequality (3.10) in (3.16) we obtain

$$
\frac{d}{d t} E_{3}(t) \leqslant\left|\int_{\Omega} v^{q-2} v w_{t} d x\right|+\frac{D_{5}}{2}\|\Delta w\|^{2}+\frac{D_{5}}{2}\left\|w_{t}\right\|^{2} .
$$

Apply the Hölder, Cauchy, Sobolev-Poincaré inequalities, and estimate (2.1) to the first term on the right hand side of (3.17) to obtain the following estimate

$$
\begin{aligned}
\beta\left|\int_{\Omega} v^{\mathbf{q}-2} v w_{\mathrm{t}} \mathrm{d} x\right| & \leqslant \beta\left\|w_{\mathrm{t}}\right\|\|v\|_{2(\mathbf{q}-1)}^{\mathbf{q}-1} \\
& \leqslant \frac{1}{2}\left\|w_{\mathrm{t}}\right\|^{2}+\frac{\beta^{2}}{2}\|v\|_{2(\mathbf{q}-1)}^{2(\mathbf{q}-1)} \\
& \leqslant \frac{1}{2}\left\|w_{\mathrm{t}}\right\|^{2}+\frac{\beta^{2}}{2} \mathrm{~d}\|\Delta v\|^{2(\mathbf{q}-1)} \leqslant \frac{1}{2}\left\|w_{\mathrm{t}}\right\|^{2}+\beta^{2} \mathrm{D}_{7}
\end{aligned}
$$

where $D_{7}=\frac{d}{2}\left(D_{1}\right)^{q-1}$. Therefore by substituting (3.18) in (3.17) we have

$$
\frac{d}{d t} E_{3}(t) \leqslant M_{3} E_{3}(t)+\beta^{2} D_{7}
$$

where $M_{3}$ is positive constant. Solving the differential inequality (3.19) we obtain the following estimate

$$
E_{3}(t) \leqslant \frac{D_{7}}{M_{3}} e^{M_{3} t} \beta^{2}
$$

Hence the proof is completed.

\section{Conclusion}

In this article, by using multiplier method, we conclude that the solution of the problem (1.1)-(1.3) describing semilinear Petrovsky equation is continuously dependent on the coefficients $a$ and $b$.

\section{Acknowledgment}

The authors would like to thank the referees for the careful reading of this paper and for valuable suggestions to improve the presentation and the style of the paper.

The precious mathematician who died in the 1999 Marmara earthquake was Professor Eyüp Sabri TÜRKER, we remember him with mercy.

\section{References}

[1] G. N. Aliyeva, V. K. Kalantarov, Structural stability for FitzHugh-Nagumo equations, Appl. Comput. Math., 10 (2011), 289-293. 1

[2] N. E. Amroun, A. Benaissa, Global existence and energy decay of solutions to a Petrovsky equation with general nonlinear dissipation and source term, Georgian Math. J., 13 (2006), 397-410. 1

[3] A. O. Çelebi, V. K. Kalantarov, D. Uğurlu, Structural stability for the double diffusive convective Brinkman equations, Appl. Anal., 87 (2008), 933-942. 1

[4] A.O. Çelebi, K. Gür, V. K. Kalantarov, Structural stability and decay estimate for marine riser equations, Math. Comput. Modelling, 54 (2011), 3182-3188. 1

[5] J. Chen, Y. Jin, Continuous dependence on a parameter for classical solutions to first-order quasilinear hyperbolic systems, (Chinese) J. Fudan Univ. Nat. Sci., 39 (2000), 506-513. 1

[6] W.-Y. Chen, Y. Zhou, Global nonexistence for a semilinear Petrovsky equation, Nonlinear Anal., 70 (2009), 3203-3208. 1 
[7] M. G. Coclite, A. Favini, G. R. Goldstein, J. A. Goldstein, S. Romanelli, Continuous dependence in hyperbolic problems with Wentzell boundary conditions, Commun. Pure Appl. Anal., 13 (2014), 419-433. 1

[8] X.-S. Han, M.-X. Wang, Asymptotic behavior for Petrovsky equation with localized damping, Acta Appl. Math., 110 (2010), 1057-1076. 1

[9] E. S. Huseynova, On behaviour of solution of Cauchy's problem for one correct by Petrovsky equation at large time values, Trans. Natl. Acad. Sci. Azerb. Ser. Phys.-Tech. Math. Sci., Math. Mech., 26 (2006), 85-96. 1

[10] B. A. Iskenderov, E. S. Huseynova, Estimation of the solution to Cauchy problem for a correct by Petrovsky equation, Trans. Natl. Acad. Sci. Azerb. Ser. Phys.-Tech. Math. Sci., Math. Mech., 29 (2009), 61-70. 1

[11] G. Li, Y. Sun, W.-J. Liu, Global existence, uniform decay and blow-up of solutions for a system of Petrovsky equations, Nonlinear Anal., 74 (2011), 1523-1538. 1

[12] A. V. Perjan, The continuous dependence of solutions of hyperbolic equations on initial data and coefficients, Studia Univ. Babeş-Bolyai Math., 37 (1992), 87-111. 1

[13] F. Tahamtani, A. Peyravi, Global existence, uniform decay, and exponential growth of solutions for a system of viscoelastic Petrovsky equations, Turkish J. Math., 38 (2014), 87-109. 1

[14] F. Tahamtani, M. Shahrouzi, Existence and blow up of solutions to a Petrovsky equation with memory and nonlinear source term, Bound. Value Probl., 2012 (2012), 15 pages. 1

[15] M. Yaman, Ș. Gür, Continuous dependence for the damped nonlinear hyperbolic equation, Math. Comput. Appl., 16 (2011), 437-442. 1

[16] Y. C. You, Energy decay and exact controllability for the Petrovsky equation in a bounded domain, Adv. in Appl. Math., 11 (1990), 372-388. 1

[17] Y. C. You, Boundary stabilization of two-dimensional Petrovsky equation: vibrating plate, Differential Integral Equations, 4 (1991), 617-638. 1 